fantastic; and, above all, of its pilots, that with the skill and technique peculiar to their craft they shall combine a practical working knowledge of structure and of machinery, of instruments and "wireless," of meteorology and of navigation, which in other professions would be the province of different specialists. And this universality, as might be expected, is no less characteristic of the appeal which aeronautics has made to the man of science, who provides it with fresh data; so that it is not surprising to find that the single committee of pre-war days has been compelled to adopt a policy of devolution, and that special subcommittees have been formed to deal with problems of such different scope as "scale effect," the investigation of accidents, internal-combustion engines, light alloys, meteorology, atmospheric electricity, and new inventions.

The report abounds with indications of fields in which further research is needed, and there seems every reason to believe that this research will be prosecuted with equal success under the auspices of the newly constituted Aeronautical Research Committee. We learn with satisfaction that the demand for the earlier technical reports has been vigorous enough to justify the printing of a complete second edition, since an opportunity is thus given for inserting much more complete cross-references than were possible when they first appeared. It is, perhaps, a matter for some little regret that a more definite lead has not been given in this direction by the present report. We imagine that any reader whose interest in the subject extends to the detailed reports of the several sub-committees would wish to have such references to individual papers and their authors as will enable him to find additional information on any special point; moreover, an account so detailed, and yet empty of names and references, may fail to impress what we believe is the secret of British supremacy in aeronautics: that our official Committee has interpreted its functions as being advisory rather than executive, and has endeavoured to assist, coordinate, and encourage research rather than to originate and control it.

No useful end would be served, and perspective would be lost, by abstracting from these excellent and thoroughly condensed reports. Their range is verv wide, extending from complete investigations, on both the practical and theoretical sides, of such complex evolutions as "spinning" to researches on the properties of light alloys, the transmission of heat from rough and smooth surfaces to passing currents of air. the conditions leading to discharge of atmospheric electricity from kite-balloons and cables, and the best shape for parachutes. Few, we believe, will read these pages without discovering some points of contact between aeronautical science and their own particular field of investigation.

Mention should be made of the very interesting table of comparative performances of British aircraft which is included as an appendix.

\section{Climatic Cycles and Tree-growth.}

PUBLICATION No. 289 of the Carnegie Institution of Washington is devoted to Prof. Douglass's study of the annual rings of trees in relation to climate and solar activity. When the late Prof. Lowell was seeking an ideal climate for his observatory, with the view of studying the planet Mars, he chose the dry region of Flagstaff, Arizona, on account of its low rainfall and high proportion of clear skies. While Prof. Douglass was at the Lowell Observatory it occurred to him that variations in solar activity might have a measurable effect on treeNO. 2644 , VOL. IO5] growth, since the latter was probably dependent on rainfall, and rainfall might very likely be affected by solar activity. He began by studying the yellow pines of those arid regions, arguing that a very dry climate should be the best for such an investigation. $\mathrm{He}$ soon found that the intimacy of the connection between the width of the annual tree-rings and the rainfall, when the latter was known, was far closer than he had dared to hope, and he pushed further afield, examining tree specimens and fossils in European collections as well as in other districts of America.

Considerable labour was involved in the interpretation of the various appearances of the rings, the red tissue that denotes the close of a period of growth. The year starts in the autumn. With normal winter snow and spring and summer rainfall growth continues throughout until the autumn, and a ring of normal width is produced. If winter snow is deficient and spring rain also scanty, a narrow ring is produced, closing prematurely without waiting for summer rain. An intermediate condition is shown when winter snow is deficient and the spring drought is not so severe; red tissue begins to form, but growth starts again, and the result is a double ring for the year.

The author considers that five trees in a group give a trustworthy result in general, though in a very dry district like Arizona two might suffice. The only district where five trees failed to give a satisfactory crossidentification was a rugged region near Christiania, in Norway.

The Flagstaff record is complete from A.D. I 385 , but among the sequoias of California stumps are in existence dating back more than three thousand years. Some of these sequoias grew on hillside slopes, and others in basins where plenty of moisture is found at all times. The latter are unsuitable for investigation, and the author calls them "complacent," as they show practically no variation in the annual growth. The others he calls "sensitive," as they have to depend upon snow melting down the slope and upon rain as it comes, not being provided with any storage such as that found in the basins. Some specimens, including the oldest of all, showed signs of a change in environment, "complacent" in later growth but "sensitive" earlier. Prof. Huntington had previously investigated these sequoias in his search for evidence of climatic change, but his purpose was served with much less detailed measurements, ten-year periods being short enough for his unit of time. His dates agree fairly well with those of the present work. The analysis of the data for periodicities required considerable accuracy in the method employed, and ultimately led to the adoption of the "automatic optical periodograph," of the construction and application of which full details are given.

Practically all the groups of trees investigated show the sun-spot cycle or its multiples; the solar cycle becomes more certain and accurate as the area of homogeneous region increases or the time of a tree record extends farther back; this suggests the possibility of determining the climatic and vegetational reaction to the solar cycle in different parts of the world. A most suggestive correlation exists in the dates of maxima and minima found in tree-growth, rainfall, temperature, and solar phenomena, pointing to a physical connection between solar activity and terrestrial weather. There is a very important point discussed under the title of "Meteorological Districts." It is essential to restrict any such district for this purpose to one in which homogeneous weather conditions are found. Clearly, if one set of conditions makes one district wet and a neighbouring district 
dry, these cannot be lumped together for correlation purposes, as the whole effect will be masked. We are reminded of the sun-spot maximum of 1893 , which was associated with great heat in England and France, but was exceptionally cold in America and other parts of the world. This limitation of districts may not, as the author recognises, be the same for short periods as for long ones, but he finds the major characteristics in mountain regions very much alike over distances of fifty or sixty miles, and relies upon the evidence of the trees themselves for the demarcation of the districts.

One other small difficulty Prof. Douglass has met in an ingenious manner. It is often noticed that such an element as rainfall, when expressed as departure from the mean, as it must be in correlation problems, is arithmetically lacking in symmetry, since the defect can only be roo per cent. at most, while excess can be very much larger. Geometrically, this can be avoided by using a logarithmic scale, but this flattens the variation very much. Prof. Douglass's device is to leave the deficient amounts unaltered, but in the case of excessive falls to invert the fraction and measure upwards from the normal. Thus a rainfall of twice the normal is indicated by a point just so far above the normal line as the point indicating a rainfall of half the normal is below it. The symmetry is not perfect, as, of course, no possible wetness can give a point corresponding to zero rainfall, but the method is convenient in places where zero rainfall in the unit period is unknown.

W. W. B

\section{The Interferometer in Physical Measure- ments. ${ }^{1}$}

AOURTH volume describing the researches of A Prof. Carl Barus with interferometers has recently been issued. The classical work of Fizeau, who applied interference methods to the determination of expansion coefficients, directed attention many years ago to the possibility of the kind of work which has been so well developed by Michelson and others, and in the present series of papers Prof. Barus seeks to develop the methods of application of the interferometer to a somewhat wide range of physical measurements. These include spherometer measurements, elastic deformation of small bodies, elongations due to magnetisation, pressure variation of specific heat of liquids, and even electrodynamometry. The remainder of the volume deals with various modifications of the interferometer methods and with certain gravitational experiments.

Doubtless such an investigation of methods will be useful to workers in any of the foregoing fields, but so far as a first impression is to be trusted it would appear that the main interest has lain in the method rather than in any results which have been attained.

In order to study the motion of a contact lever, it may be made to carry two small mirrors reflecting normally two beams which are afterwards caused to interfere. Any rotation of the lever obviously causes a difference of path, which appears in the shifting of the easily recognisable and distinctive central "achromatic" interferometer fringes, such motion being measured by a plate micrometer or "graticule" in the observing telescope.

The two mirrors form the limbs of a " $T$ " piece, which is pivoted about a hinge at the end of the foot. One limb ends in a contact pin which abuts against the surface, the motion of which is to be measured.

In such circumstances Prof. Barus estimates the

1 "Displacement Interferometry by the Aid of the Achromatic Fringes." Part iv. By Prof. Carl Barus. (Carnegie Institution of Washington, I919.) NO. 2644, VOL. IO5] limiting sensitiveness to be $33 \times 10^{-6} \mathrm{~cm}$., or perhaps even a third of this amount, but it should not be forgotten that the very simple interferometer system of an optical test-plate has a sensitiveness of about a quarter wave-length, say $12 \times 10^{-6} \mathrm{~cm}$., and this without a doubtful hinge and another contact. The contact lever can, of course, deal with non-specular surfaces, but to use it as a spherometer for a glass lens seems quite needless. Naturally, an apparatus of this nature is excellently adapted to such a problem as that of investigating the changes of length of a magnetised rod, and, although no very novel resuits are obtained, the investigation has been comparatively easy, and the method is well adapted for demonstration.

Suitable self-adjusting interferometers, such as are described in chap. vii., ought to find an increasingly useful place in the physical laboratory, and students should be taught the practical use of such instruments and their modifications. There is too great a tendency to treat an interferometer as a piece of apparatus sacred to one or two highly specialised purposes, but with little more than a few pieces of good plane parallel glass a set of instruments can be made up which should be of the greatest use in teaching and research.

One could wish, perhaps, that some one problem had been attacked and solved thoroughly. The curiously unfinished nature of the work is disappointing, but we must conclude that the method is the chief object. As regards the text, the descriptions are clear and praiseworthy, but the diagrams are both inadequate and unsatisfactory. L. C. M.

\section{Canvas-destroying Fungi.}

WHEN men again began to take to their tents at the outbreak of war, many noticed that dark brown and black spots, frequently of a diamond shape, were not uncommon on the canvas. Small, surreptitiously acquired bits began to be scattered around for information as to the identity of the moulds causing the rot. Now it is very surprising that so little work has been done on canvas-destroying fungi. That canvas is liable to suffer from moulding seems generally to be known, judging from the fact that any material likely to get wetted is usually "cutched." Shortly before the war aircraft workers began to interest themselves in the fungi concerned in the damage, but it was not until war. broke out that one realised the extent of the destruction of sails, tents, etc., by these organisms.

Major W. Broughton-Alcock, in the Journal of the Royal Army Medical Corps for December last, gives a short account of investigations carried out by him in Malta, Italy, and (in conjunction with Miss A. Lorrain Smith) at the Natural History Museum. In Malta attention was soon attracted to the rapid spotting and destruction of tentage-awnings last there only about a vear. The investigators found that the principal agents of destruction of cotton- and flaxmade canvas are Macrosporium and Stemphylium. The latter is the more prevalent in Malta, and could be isolated by exposing culture plates to the air. The colours of the spots on canvas correspond to the colours seen in cultures, being first brown and then black. The variation in the colour of the spots, especially noticed in flax-made and more resistant canvas, was found to be due to other fungi in association with the above genera--Septoria, Alternaria, Helminthosporium. Chætomium, Exosporium, Penicillium, Oospora, Torula, Saccharomyces, and yellow pigment-forming and other air-borne bacteria. Though 